\title{
MOLECULAR BASIS OF COOPERATIVITY IN VERTEBRATE MUSCLE THIN FILAMENTS
}

\author{
by \\ P.W. BRANDT ${ }^{\prime \prime}$, M.S. DIAMOND", B. GLUCK", M. KAWAI") and F. SCHACHAT ${ }^{2 \prime}$ \\ "Columbia University, Department of Anatomy and Cell Biology, \\ 630 West 168th Street, New York, N.Y. 10032, USA \\ ${ }^{2)}$ Duke University, Department of Anatomy, North Carolina, USA
}

Keywords: Cooperativity, thin filaments, troponin, tropomyosin, regulation

\begin{abstract}
Extraction of as little as one troponin $C$ per regulatory strand on a thin filament reduces the slope of the $\mathrm{pCa} /$ tension relation thus the regulatory units along a thin filament of rabbit psoas fibers are linked cooperatively so that a thin filament activates as a unit. This explains why the $\mathrm{pCa}$ /tension relation in skinned fibers has a slope much higher than can be expected by binding of $\mathrm{Ca}^{2+}$ to one regulatory unit (BRANDT et al, 1980; BRANDT et al. 1982). These results also show that troponin $C$ is the only myfibrillar component responsible for calcium sensitivity in psoas muscle.
\end{abstract}

\section{INTRODUCTION}

There is much evidence that the regulatory units of the thin filament of vertebrate muscle cooperatively activate a length of filament when $\mathrm{Ca}^{2+}$ is bound to a subunit, troponin $\mathrm{C}(\mathrm{TnC}) . \mathrm{A}$ striking physiological demonstration of this is the high slope of the relation between $\mathrm{Ca}^{2+}$ and tension which, in the psoas muscle of the rabbit, has a Hill coefficient $\left(n_{H}\right)$ between 5 and $6(3,4$, 26), although there are only two $\mathrm{Ca}^{2+}$ binding sites on $\mathrm{TnC}$ that regulate contraction (31). One way to explain the large $n_{H}$ is to postulate cooperative interaction between regulatory units on a thin filament.

The thin filament can be considered as two strands of actin with two regulatory strands wound together into a double helix. The regulatory strands are made up of troponin and tropomyosin units which overlap end to end (37). Strands contain 26 units, assuming each unit to be $38.5 \mathrm{~nm}$ long and a thin filament $1 \mu \mathrm{m}$ long. Each unit consists of one tropomyosin and one troponin, the latter composed of three subunits called $\operatorname{TnC}, \operatorname{TnI}$ and $\operatorname{TnT}(15,21)$. Each unit overlies a sequence of 7 actins and, to a first approximation, it regulates only those actin molecules. Two levels of cooperativity have been proposed (19); the first, an all or none activation of the 7 actins, and the second, a linkage between contiguous regulatory units.

The steric hindrance model of the control process proposes that a regulatory unit physically blocks the myosin binding sites on actin strands in the absence of calcium $(18,21,29)$ and when $\mathrm{Ca}^{2+}$ is bound to $\mathrm{TnC}$, these units move sufficiently to allow myosin to bind. This model is now being challenged (10) by evidence showing cooperation between molecular components is more likely than physical blocking $(9,35)$.

To examine the role of the regulatory proteins in cooperativity, we extract $\mathrm{TnC}$ and replace it with purified $\mathrm{TnC}$. We measure the effects of these manipulations by determining the $\mathrm{pCa} /$ tension relation $\left(-\log \left[\mathrm{Ca}^{2+}\right] /\right.$ tension relation). We also examine "natural" experiments where different muscle fiber types have differently 


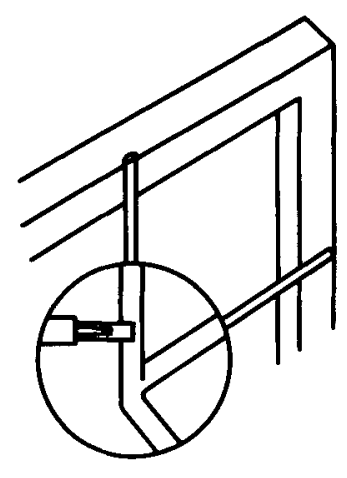

C
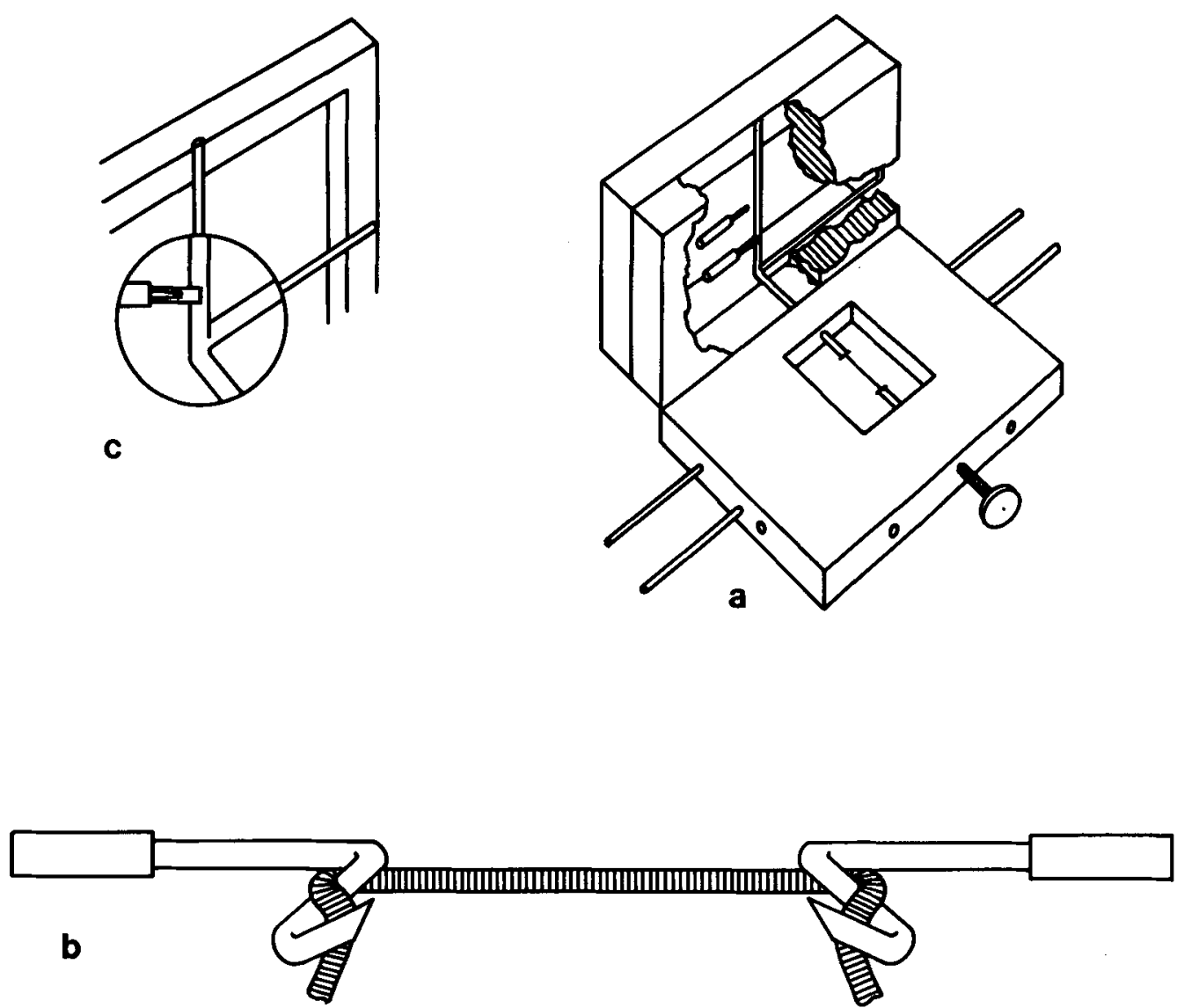

Figure 1. The experimental chamber (a) has two stainless steel tubes which end in muscle hooks, extending into the specimen well from the tension transducer (box behind the chamber) and the length adjustment screw (projecting to the front of the chamber). These enter through teflon lined holes, and the clearance between the steel tubes and the teflon is sealed with vaseline. Other stainless steel tubes which enter the chamber through the side walls are used to cary fluid into and out of the specimen well. These make tight contact with the chamber walls so that heat transfer is maximized. Water from a regulated batch is circulated through the walls of the chamber to keep the temperature constant. The muscle fiber is mounted by wrapping it around the hooks as shown in (b). In (c) an enlargement of the contact between the strain gauge (AS Akers $\neq A E 801$, Horten, Norway, Brandt et al. (2)) and the lever to the muscle hook is shown.

shaped $\mathrm{pCa}$ /tension curves correlated with variants of the regulatory proteins and myosin.

\section{METHODS}

\subsection{Skinned muscle preparation}

Strips of muscle about $1-2 \mathrm{~mm}$ in diameter and $30 \mathrm{~mm}$ in length are separated lengthwise from whole muscles of rabbits killed by $\mathrm{CO}_{2}$ inhalation and decapitation. These strips are tied at two places to small wooden sticks, the fiber ends cut free from the rest of the muscle, and the chemically skinned in a saline containing (in mM); 5 EGTA, 2 MgATP, 5 ATP, $130 \mathrm{~K}$ propionate, 5 imidazole ( $\mathrm{pH} 7.0$ ) for 48 hours at $2{ }^{\circ} \mathrm{C}(22,39)$. After skinning, some of the fiber bundles are kept refrigerated in this solution and used in experiments up to 2 weeks; the rest are kept at $-20^{\circ} \mathrm{C}$ in an iso-ionic glycerol saline (skinning saline in $6 \mathrm{M}$-glycerol). By comparison refrigerated fibers to those kept in the freezer, we eliminate the possibility that either glycerol or freezer storage significantly alters the preparation. Single fibers are isolated from the stock 
bundle. For a detailed report on the preparation and ultra-structure see EASTwood et al. (12).

\subsection{Muscle chamber}

The muscle chamber (Figure la) is made of an aluminium block painted with inert plastic. It has an inside capacity of $0.5 \mathrm{ml}$. Stainless steel (ss) tubes from the length adjustment device and force transducer assembly, respectively, enter the chamber through air gaps in teflon collars. Although saline does not readily leak through these collars, a small amount of vaseline is applied at the outer ends of the gaps. The air trapped in the collars prevents the vaseline from touching the solution. The chamber is heated by a focused lamp and a circulation system carries temperature regulated water through channels in the chamber walls. Thermistors are combined in a feed back network to maintain the temperature at the desired level $\left(20 \pm 0.1^{\circ} \mathrm{C}\right)$. The saline is constantly mixed by a vibrating stirrer to minimize local heterogeneity in ionic concentrations ("unstirred layer"; 28) and in temperature. Other ss tubes forced through holes in the walls of the chamber carry solutions into or out of it. A small slit in the side of the hollow ss stirrer is used to suck excess solution off at the surface. The constant agitation of the orifice keeps a meniscus from forming so that solution withdrawal stops at a reproducible level (volume) in the chamber.

\subsection{Fiber mounting procedure}

A segment of a single fiber $6-10 \mathrm{~mm}$ long is dissected from a stock bundle and transferred to the experimental chamber filled with the relaxing saline. The ends of the preparation are wrapped around bent ss wires (Figure $1 b$ ) and wedged under small clips (3). The sarcomere length is adjusted to $2.5-2.6 \mu \mathrm{m}$ as measured by the separation of the 1 st order diffraction bands on a reticon array output to an oscilloscope. (The light source is a He-Ne laser.) Muscle at this length is defined as $L_{0}$. It is approximately $10 \%$ above the slack length and corresponds to the peak of the active length-tension diagram in mammalian skeletal muscles.

\subsection{Force transducer}

The force transducer (Figure $1 \mathrm{c}$ and (3)) has low compliance ( $2 \mathrm{~nm} / \mathrm{dyn})$, high sensitivity $(0.1$ dyn change can be detected), high stability, and little drift $\left(0.005 \% /{ }^{\circ} \mathrm{C}\right)$. The resonant frequency is $2 \mathrm{KHz}$ with a muscle clamp attached.

\subsection{Automated apparatus}

This consists of a Pro-log PLS 441 microprocessor (Pro-log Corp., Monterey, Ca.), a paper tape punch, pumps, valves, and a hexadecimal keyboard used to input the experimental parameters, which in turn select the program modules, Figures 2 and 3 . The tension preamplifier output goes to a digital voltmeter that ranges \pm 2000 millivolts ( $1 \mathrm{dyn} / \mathrm{mv}$ ). Four digits (binary coded decimal) obtained from the voltmeter are input separately to the processor as well as time (hours, minutes, and seconds) from a clock chip. Digital outputs of the processor control valves and high precision pumps driven by stepping motors. For a visual record and as a backup, tension, temperature, and timing signals are also traced on a 2-channel Brush pen recorder (not shown) equipped with two even markers.

The experimental control program is modular and includes 8 separate general purpose routines. Many of these routines end by calling a subroutine in which the system waits until the tension becomes stable and then punches the steady state data on paper tape. Examples of the modular routines include: (i) Change the bathing saline for one stationed behind a specified valve. (ii) For $\mathrm{N}$ repeats, pump in a specified volume of saline with a given pump and go to the subroutine to wait for the steady tension state; then punch the data. This is the main routine for collecting a $\mathrm{pCa}$ /tension curve. Because the chamber volume is kept constant as the solution is pumped in, the composition of the saline in the chamber can be calculated (solution section below). (iii) Wait $M$ seconds before proceeding. $M$ is chosen by starting with large values and observing where the slope of the time course curve becomes essentially flat. $M$ is then decreased to this value. (iv) Punch an "end of curve" code on the paper tape.

At the time of an experiment, a series of two hexadecimal codes is entered on the keyboard. 


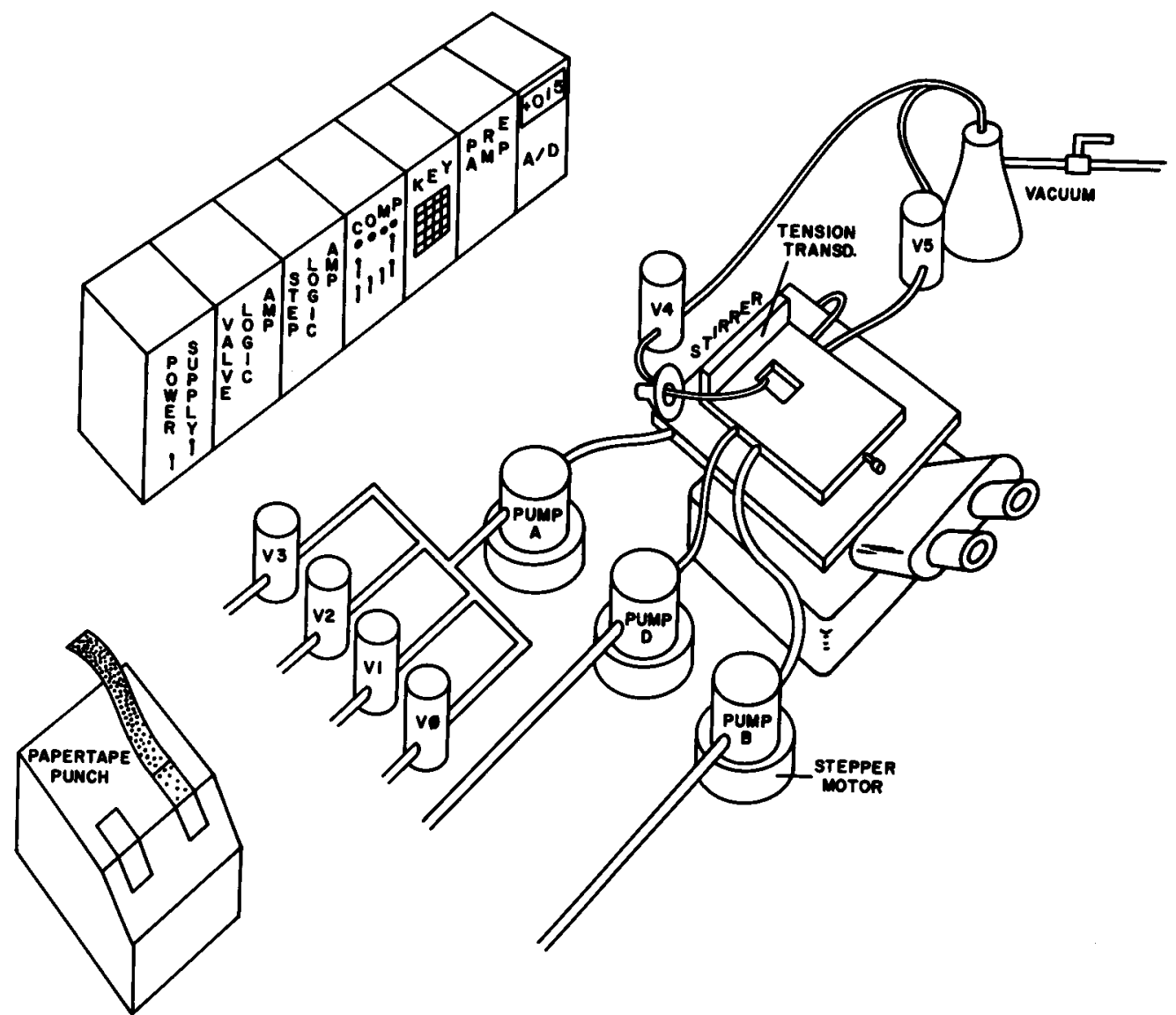

Figure 2. A cartoon of the apparatus which automatically collects pCa/tension curve data. An inverted microscope is the base for the muscle chamber. The "stirrer" is a vibrating tube driven by a small magnetic motor at $60 \mathrm{~Hz}$ and an orifice in the side of the tube near the tip is used to aspirate fluid from the well. Because it is vibrating rapidly, no meniscus forms at the orifice and aspiration stops at a fixed level of fluid in the chamber. Four valves feed solution to one pump which is used to change the fluid in the chamber. Two other pumps (Fluid Metering Corp., Oyster Bay, LI, N.Y.) meter solution directly into the chamber.

The determine the program module and the control data for the module, such as specifying the valve, pump, volume, timing, etc. It takes about 6 codes to generate a $\mathrm{pCa} /$ tension curve, but commonly 12 or more are entered - one set for a control curve and one for an experimental curve. Because 512 codes can be stored in the processor, a very complex sequence can be automated. Once a fiber is mounted, the solutions placed in the appropriate reservoirs, and the protocol keyed in, the experiment proceeds without further intervention by the investigator. Automation eliminates various kinds of errors, such as timing errors, bias in estimating the steady tension, baseline adjustment and truncation errors on the pen recorder, and, most importantly, chart reading errors.

\subsection{Solutions}

The standard pCa 8 "relaxing" solution contains ( $\mathrm{Na}$ salts in $\mathrm{mM}$ ): 9.8 EGTA, 0.2 CaEGTA (ethylene glycol bis(-aminoethylether)N,N,N'N'-tetraacetic acid), 5 MgATP, $5 \mathrm{Na}_{4} \mathrm{ATP}, 7.5 \mathrm{NaPi}$ (phosphate), 40.1 $\mathrm{Na}$ propionate, 17.2 $\mathrm{Na}_{2} \mathrm{SO}_{4}, 10$ MOPS (morpholinopropane sulfonic acid) at $\mathrm{pH}$ 7.00. EGTA and all forms of ATP are removed to 


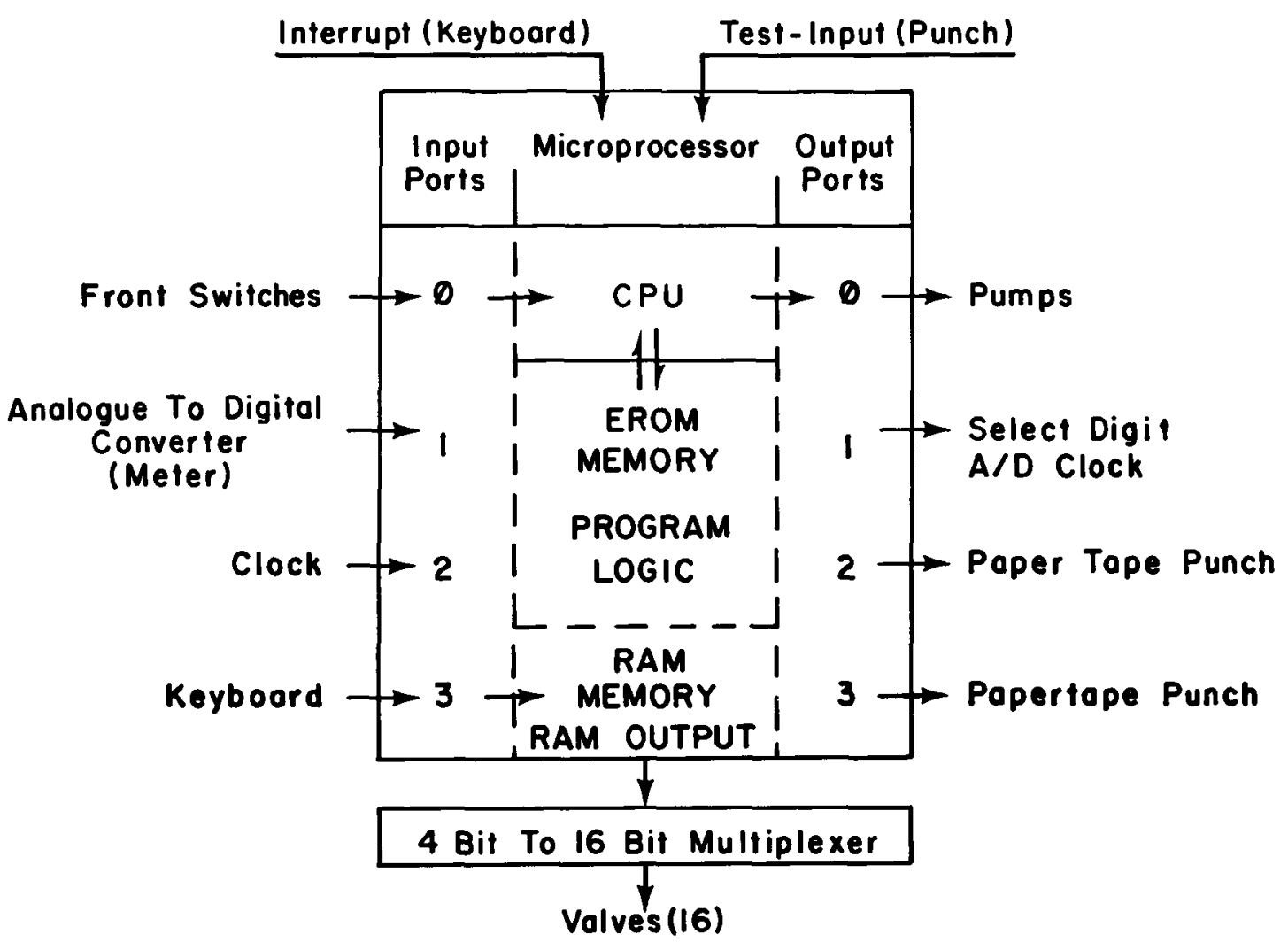

Figure 3. The general logic of the microprocessor (Intel 4040) control is shown in block form. Each 4 bit input or output port is numbered $\mathbf{0 - 3}$. The RAM (read/write memory) port is at the bottom of the diagram. At the top one bit inputs are for the interrupt line from the keyboard and the status line from the paper tape punch. Most of the program logic is stored in the EROM (erasable/programmable read only memory) and at the time of an experiment a control sequence is input from the keypad and stored in RAM. The control sequence selects a series of options build into the program logic. After each solution change, most steps in the sequence include logic to wait for steady tension, then punch the steady state values on the paper tape. The data on paper is input to a large minicomputer for data reduction, curve fitting and plotting. See text for further details.

make the "wash" solution: $195 \mathrm{mM}-\mathrm{Na}$ propionate and 10 MOPS. This solution is applied prior to the rigor solution to remove all but trace amounts of substrate. "Rigor" solution contains $10 \mathrm{~mm}$ total EDTA (CaEDTA:EDTA ratio to obtain pCa 8), 10 MOPS and 165 Na propionate. By chelating the $\mathrm{Mg}$ the last trace of substrate is rapidly removed so little tension is generated. The "activating" solutions contain: $10 \mathrm{~mm}$ total EGTA (CaEGTA:EGTA ratio according to the $\left.\mathrm{pCa}=-\log \left[\mathrm{Ca}^{++}\right]\right), 5 \mathrm{MgATP}, 5$ free ATP, 10 MOPS, 7.5 $\mathrm{NaHPO}_{4}$, and $\mathrm{Na}$ propionate to adjust the final solution to our standard ionic strength $(.200)$. The concentrations of multiva- lent ionic species are calculated after solving the multiple equilibria of two metals $(\mathrm{Ca}, \mathrm{Mg})$ and two ligands (EDTA or EGTA and ATP), by using the following apparent association constants (log values at $\mathrm{pH} 7.00$ ): MgEDTA 5.4, CaEDTA 7.3, CaEGTA 5.28, MgEGTA 1.6, CaATP 3.70, MgATP 4.00. All experimental solutions are adjusted to $\mathrm{pH} 7.00 \pm 0.01$, and the temperature is maintained at $20.0 \pm 0.1^{\circ} \mathrm{C}$.

We make the series of $\mathrm{pCa}$ solutions directly in the muscle chamber by injecting a pCa 4.865 stock saline into the chamber (filled initially with pCa 8) while keeping the solution volume in the chamber $\left(v_{0}\right)$ constant. Applying the following 
Tension

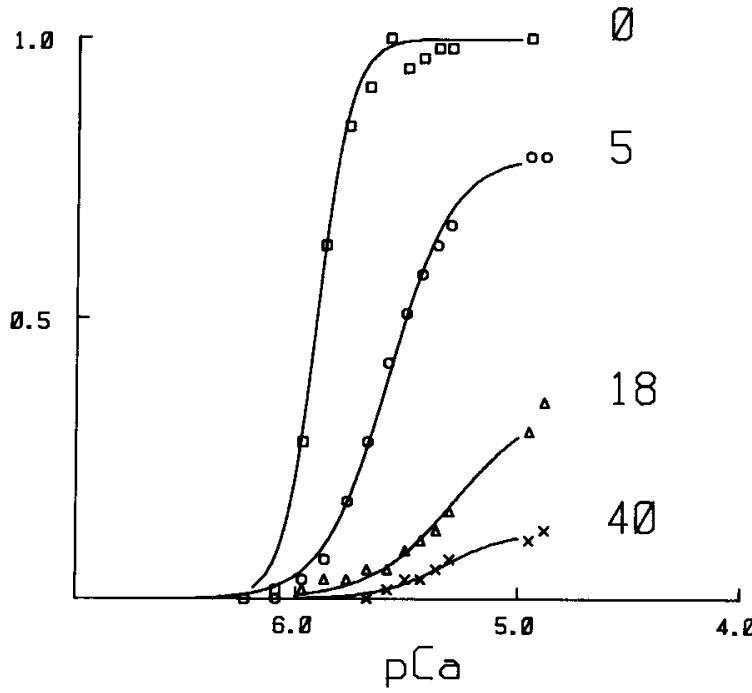

Figure 4. Tension relative to that for a control fiber is plotted against the $\mathrm{pCa}$ for three fibers exposed to a solution designed to extract $\mathrm{TnC}$ for the times indicated to the right of each curve. The fibers were all $70 \mu \mathrm{m}$ in diameter, dissected from the same bundle and run the same day. The figure shows that extraction of $\mathrm{TnC}$ results in a progressive loss in slope and maximum tension of the $\mathrm{pCa}$ /tension relation with time of extraction. The reduction in slope is not accompanied by an obvious change in threshold $\mathrm{Ca}^{2+}$, therefore there is also a progressive shift of the $\mathrm{pK}$ to lower values (see Table I). equation, the pCa can be calculated at the volume $(v)$ of injected stock $\left(C_{s}\right)$.

$$
\mathrm{C}_{\mathrm{v}}=\mathrm{C}_{\mathrm{s}}+\left(\mathrm{C}_{\mathrm{o}}-\mathrm{C}_{\mathrm{s}}\right)_{\mathrm{e}}-\mathrm{v} / \mathrm{v}_{\mathrm{o}} \quad \text { (1) }
$$

When we start with pCa 8 in the chamber and inject pCa 4.865 we can obtain a pCa series from about pCa 7 to pCa 4.9, then if necessary we can switch to a pCa 4 stock and continue the series (see ref. 3 and 4 for further details on the serial dilution method and verification of its accuracy).

Starting solutions are all calculated by a computer program that prints the volume of each stock solution to add to obtain a specified pCa. The program keeps both the ionic strength and the monovalent cation concentration constant. The approach of specifying an outcome is much simpler than calculating the outcome of a given mix of stocks. The general technique is described in BRANDT et al. (1).

\subsection{Data reduction}

The $\mathrm{pK}(\operatorname{logK})$ is the midpoint of the $\mathrm{pCa} / \mathrm{ten}$ sion curve and $n_{H}$ is the Hill coefficient ( $n$ in equation 2) calculated by fitting the data by a least squares program (3) to the following form of the Hill cooperativity equation:

$$
\mathrm{P} / \mathrm{P}_{\mathrm{o}}=\left\{\left[\mathrm{Ca}^{2+}\right] \cdot \mathrm{K}\right\}^{\mathrm{n} /}\left(1+\left\{\left[\mathrm{Ca}^{2+}\right] \cdot \mathrm{K}\right\}^{\mathrm{n}}\right)
$$

\subsection{Extraction of troponin $C$}

TnC is extracted from single skinned fibers by exposing them to $5 \mathrm{mM}$-EDTA and $10 \mathrm{mM}-$

Table I.

To determine the $\mathrm{TnC}$ after various times in the extraction media, 10 to 20 single fibers were extracted and frozen until SDS gel electrophoresis was done. The gels were stained by the Coomassie method then photographed and the densities of the bands measured. Each time point is the mean of 6 different determinations. Similar dhta was obtained from silver stained gels of single fibers (Figure 9). The pK is the midpoint of the pCa/tension curve and $n_{H}$ is the Hill coefficient calculated by fitting the data to equation 2 . The number of fibers refers to all the rows except the TnC (\%) row. The SEM's are listed after each value in the table.

\begin{tabular}{llllllll}
\hline & \multicolumn{8}{c}{ Minutes extracted } \\
\cline { 2 - 8 } & 0 & 2 & 5 & 10 & 20 & 30 \\
\hline Parameter & $100 \pm 4$ & $77 \pm 3$ & $69 \pm 8$ & $40 \pm 6$ & $31 \pm 2$ & $18 \pm 2$ \\
TnC (\%) & $100 \pm 8$ & $76 \pm 8$ & $40 \pm 6$ & 28 & \pm 6 & & \\
Tension (\%) & $6.3 \pm 0.5$ & $3.3 \pm 0.2$ & $2.1 \pm 0.1$ & $2.0 \pm 0.3$ & \\
$\mathrm{n}_{\mathrm{H}}$ & $5.97 \pm 0.02$ & $5.65 \pm 0.03$ & $5.38 \pm 0.05$ & $5.21 \pm 0.08$ & \\
pK & 11 & 13 & 12 & 8 & & \\
Number fibers & 11 & & & & & \\
\hline
\end{tabular}


Table 11.

Physiological data on fibers from several fast twitch glycolytic muscles of the rabbit. $N=$ number of fibers tested. $n_{H}$ and $p K$, Hill equation (2) coefficients obtained as described in methods. $F_{T n T r}$ is the fraction $\operatorname{TnT}_{11}:\left[\operatorname{Tn} T_{15}+T_{n} T_{2 f}\right]$, where $T n$ is troponin. $F_{(\alpha) T m}$ is the fraction $(\alpha) T m:[(\alpha) T m+(\beta) T m]$, where $T m$ is tropomyosin.

\begin{tabular}{lrllll}
\hline Muscle & $\mathrm{N}$ & $\mathrm{pK}$ & $\mathrm{n}_{\mathrm{H}}$ & $\mathrm{F}_{\mathrm{TnT/ \textrm {f }}}$ & $\mathrm{F}_{(\mathrm{a}) \mathrm{Tm}}$ \\
\hline Psoas & 23 & 5.85 & 5.33 & 0.13 & 0.76 \\
Plantaris & 5 & 5.78 & 3.7 & 0.54 & 0.55 \\
Tibialis & 18 & 5.68 & 3.1 & 0.45 & 0.58 \\
\hline
\end{tabular}

MOPS at $\mathrm{pH} 7.2$ and $30^{\circ} \mathrm{C}$. This is a modification of the procedure of Cox et al. (11). Before exposure to extracting solution, fibers are washed with the sequence, relaxing solution, wash solution, then rigor solution.

\subsection{Protein preparations}

Myofibrils were prepared as described previously $(8,33)$. Myosin was purified according to SCHACHAT et al. (33) and tropomyosin, troponin, and troponin subunits according to EISENBERG and KIELLEY (13) with the modifications described in BRONSON and SCHACHAT (8).

\subsection{Gel electrophoretic analysis}

Polyacrylamide gel electrophoresis in the presence of SDS was performed according to the procedure of LAEMMLI (23) with the ratio of acrylamide to $\mathrm{N}, \mathrm{N}$ '-methylene bis acrylamide adjusted to 27. Staining was with Coomassie brilliant blue as described in BRONSON and SCHACHAT (8).

\section{RESULTS}

The steady increase in tension in a normal skinned fiber (Figure 4, top curve) for each incremental change in $\mathrm{Ca}^{2+}$ is plotted against the $\mathrm{Ca}^{2+}$ (shown here as the $\mathrm{pCa}$ or $-\log \left[\mathrm{Ca}^{2+}\right]$ ). The $\mathrm{n}_{\mathrm{H}}$ of the curve is expected to be between 1 (no cooperativity in calcium binding) and 2 (maximum cooperativity) because it is known that only two of the four $\mathrm{Ca}^{2+}$ binding sites on $\mathrm{TnC}$ regulate contraction ( 31 ). Instead it is 5.9 for the control curve in Figure 4 and the mean is 6.3 for the series in Table I and 5.3 for the series in Table II.
Extraction of $\mathrm{TnC}$ reduces both the slope and the maximum tension of the $\mathrm{pCa} /$ tension relation. Figure 4 shows the $\mathrm{pCa} /$ tension relation for a series of fibers extracted for times from 5 to 30 minutes. The curves are not saturated at $\mathrm{pCa}$ 4.85 , the lowest $\mathrm{pCa}$ tested although the control curve (top curve) saturates by $\mathrm{pCa} 5.7$. Progressive flattening of the curve accompanies increase in extraction time and there is not much change in threshold $\mathrm{Ca}^{2+}$. In other experiments we have extended the curve to lower $\mathrm{pCa}$ (higher $\mathrm{Ca}^{2+}$, see Figure 6) and found that the tension flattens about pCa 4.

To quantify the amount of $\mathrm{TnC}$ in the fibers after various times of extraction we put batches of 10 to 20 single fibers through the procedure and prepared them as one sample for SDS gel electrophoresis. After staining with Coomassie, they were photographed and the densities of the various bands measured (Table I). To account for differences in gel loading, the density of the TnC band was normalized against the sum, $\mathrm{Tm}+\mathrm{TnT}+$ myosin light chains. Because it has been reported that light chain 2 is extractable (25) we determined that no relative extraction of this occurred before it was included in the normalization factor. The tension and quantity of $\mathrm{TnC}$ decrease roughly together with time of extraction (Table I), at least initially, then tension seems to decrease faster. The decrease in $\mathrm{TnC}$ is approximately first order with a half-time of about 8 minutes (Figure 5). $n_{H}$ decreases to limit of about 2, and the pK decreases to 5.2.

By replacing the extracted $\mathrm{TnC}$ with purified $\mathrm{TnC}$ it can be shown that the only significant correlate of the slope change is TnC loss. When extracted fibers are incubated at $20^{\circ} \mathrm{C}$ in pCa 8 relaxing solutions containing $1.5 \mathrm{mg} / \mathrm{ml} \mathrm{TnC}$ (purified by the procedure of EISENBERG and KIELLEY (13)) both the slope and the tension are 


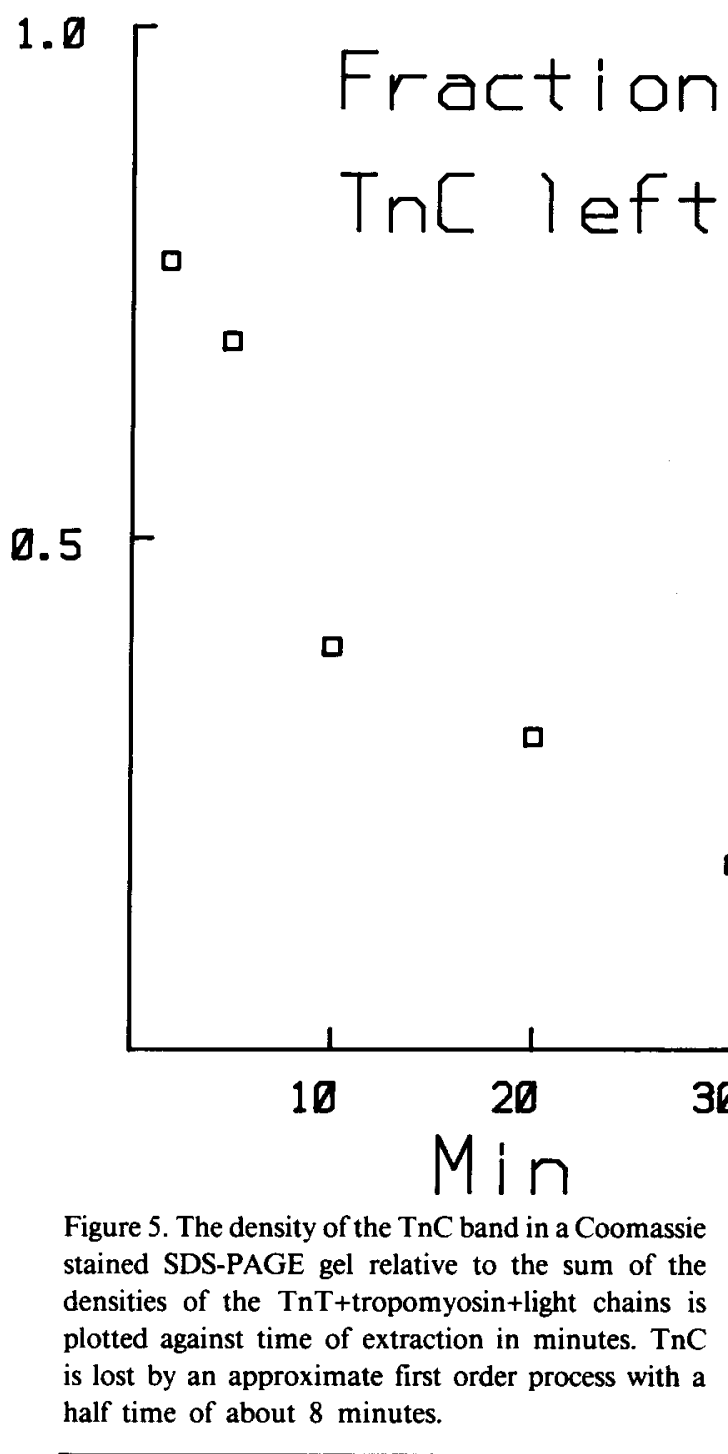

restored to near control values. The fiber in Figure 6 was first extracted for 5 minutes (lower curve), then incubated in $\mathrm{TnC}$ solution for $\mathbf{2 0}$ minutes (upper curve). Both the absolute tension and the slope of the $\mathrm{pCa} /$ tension curve decrease with extraction then increased dramaticallyon incubation in the purified $\mathrm{TnC}$ solution. Because the effect of partial extraction of $\mathrm{TnC}$ on the slope of the $\mathrm{pCa}$ /tension relation is reversed by $\mathrm{TnC}$ addition, the effect of extraction must be due to $\mathrm{TnC}$ loss rather than loss of some other component.

Gaps in the regulatory units on the thin
Tension

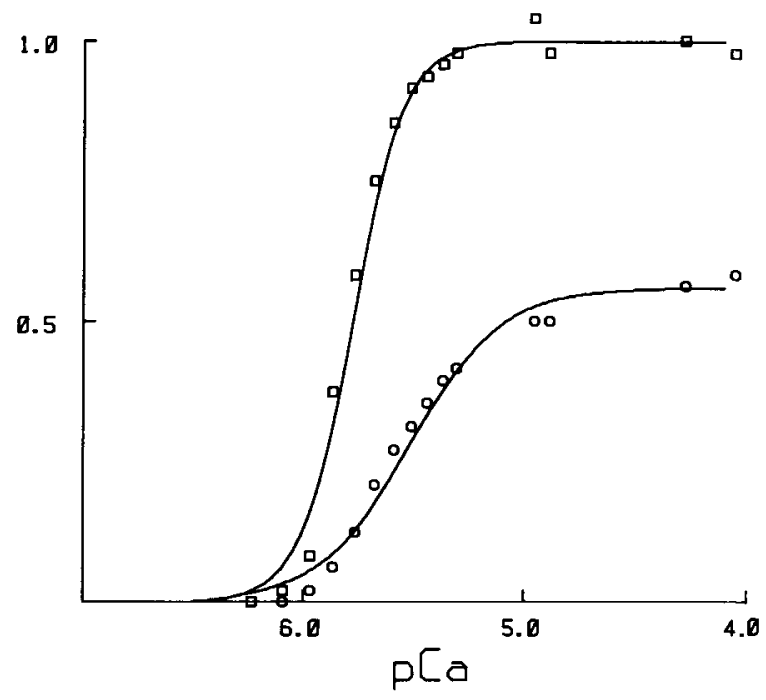

Figure 6. The fiber was first exposed for 5 minutes to a solution which extracts $\mathrm{TnC}$ (see text) then a

D $\mathrm{pCa}$ /tension curve $(O)$ determined. $\mathrm{N}_{\mathrm{H}}=2.1, \mathrm{pK}=$ 5.52 and $P_{o}=28 \mathrm{mg}$. Subsequent to this the fiber was incubated in $\mathrm{TnC}$ solution $(1.5 \mathrm{mg} / \mathrm{ml}$ in $\mathrm{pCa}$ 8 relaxing solution) for 20 minutes and the second ( $\square$ ) $\mathrm{pCa}$ /tension curve determined. $\mathrm{n}_{\mathrm{H}}=3.7, \mathrm{pK}=$ 5.78 and $P_{o}=50 \mathrm{mg}$. (Fiber C2i.10, dia. $62 \mu \mathrm{m}$ )

filament, introduced by $\mathrm{TnC}$ extraction, must break the cooperative spread of activation along the thin filament. To determine how many units in an unbroken sequence are required to generate the normal high slope, we have extracted fibers for 1 minute or less, then incubated them with $\mathrm{TnC}$ and characterized the $\mathrm{pCa}$ /tension relation after extraction and again after incubation in purified $\mathrm{TnC}$. It is difficult to quantify small losses of TnC by SDS gel so we assume that the TnC lost can be estimated from the difference in absolute tension between the extracted and the incubated curves. It is evident that the slope and tension is higher for the $\mathrm{pCa} /$ tension curve which follows incubation with $\mathrm{TnC}$ (Figure $7, \square$ ) than it is for the curve taken after 1 minute extraction $(O)$.

Extraction of even $10 \%$ of the TnC substantially reduces the cooperativity. Thus continuous sequences of intact regulatory units as long as $6,(10 \%$ of 26 is $3 \mathrm{TnC}$ subunits and 3 breaks make 4 segments) or about $1 / 4$ of normal, 
are less cooperative than the intact sequence of 26. In experiments similar to that of Figure 7, so little $\mathrm{TnC}$ is lost that the increase in maximum tension with incubation in $\mathrm{TnC}$ solution is less than $4 \%$ (about our resolution limit due to loss of maximum tension with repeat of the $\mathrm{pCa} /$ tension measurement). In such experiments (Figure 8) we find that the slope of the $\mathrm{pCa} /$ tension relation is lower in the extracted than the incubated condition. If the extracted condition is assumed to be equivalent to loss of one or less $\mathrm{TnC}$ per strand then cooperativity diminishes if the strand has one defect. Therefore, we suggest that the intact thin filament cooperatively activates as a unit.

Because we interpret these results as evidence that cooperativity is transmitted or conducted along the thin filaments, and that it depends upon the integrity of the regulatory strand, we have studied naturally occurring differences in the troponin and tropomyosin components. Earlier $(7,8,33)$ we described heterogeneity of

\section{Tension}

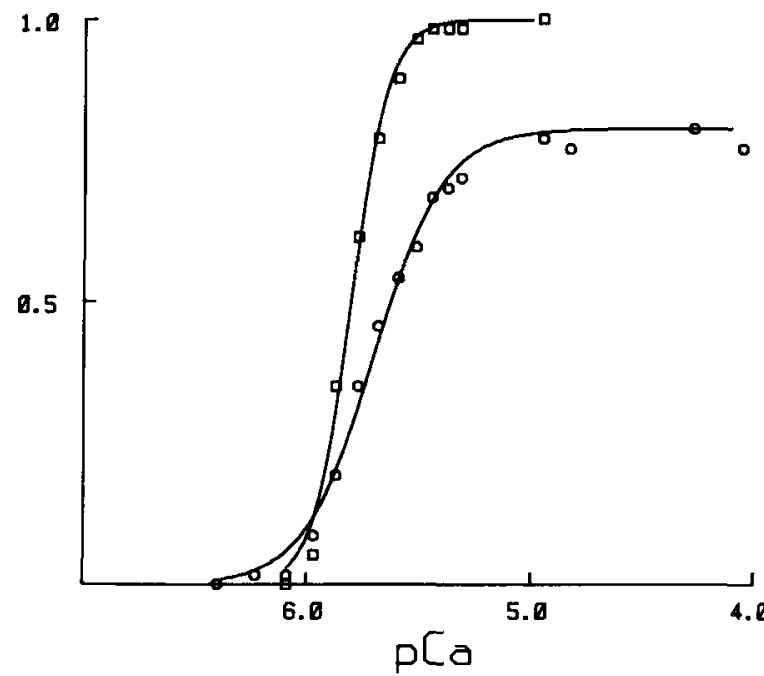

Figure 7. The fiber was first exposed to extracting solution for 1 minute before the $\mathrm{pCa} /$ tension relation determined $(O) . n_{H}=2.8, p K=5.7$ and $P_{o}=46 \mathrm{mg}$. Subsequently the fiber was incubated in $\mathrm{TnC}$ solution for 20 minutes, then the $\mathrm{pCa} /$ tension relation $(\square)$ was measured again. $n_{H}=5.2, p K=5.8$ and $P_{0}=56 \mathrm{mg}$. (Fiber C2i.23, dia. $57 \mu \mathrm{m}$ )

\section{Tension}

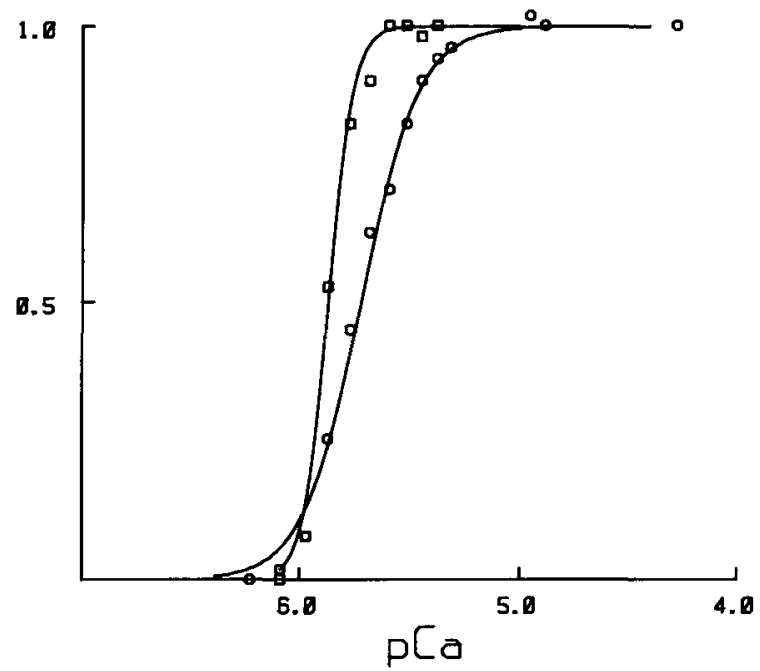

Figure 8. TnC was extracted for 1 minute then the $\mathrm{pCa}$ /tension curve was determined (O). $\mathrm{n}_{\mathrm{H}}=3.3, \mathrm{pK}$ $=5.72, P_{0}=52 \mathrm{mg}$. Next the fiber was incubated with $\mathrm{TnC}$ solution for 20 minutes and the $\mathrm{pCa}$ /tension curve against determined ( $\square$ ). $\mathrm{n}_{\mathrm{H}}=7.8, \mathrm{pK}=5.9$ and $P_{0}=51 \mathrm{mg}$. With extraction of 1 minute there is variation in the $\% \mathrm{TnC}$ extracted, and in this case so little was extracted that the curve following incubation in $\mathrm{TnC}$ showed no increase in tension. Because there is always a $5 \%$ or larger loss in tension in the second curve when two control curves are done in succession (5) (a commonly observed but not understood fatigue), we argue that the curve following incubation in $\mathrm{TnC}$ can have up to a $5 \%$ increase in activation even though no increase in tension is recorded (Fiber D2kl.23, S1 $=2.6 \mu \mathrm{m}$, dia $=57 \mu \mathrm{m}$ ).

The data of Figures 4 and $6-8$ are normalized then fitted by a least squares computer program to a form of the Hill equation (Equ. 2). The two fitting parameters, $n_{H}$ and $p_{K}$, are used in this equation to generate the smooth lines fitted to the data points.

troponin and tropomyosin in fast and slow fibers. Figure 9 shows the extent of that heterogeneity in myofibrillar preparations. Specifically there is variation in the ratio of the $(\alpha)$ and $(\beta)$ tropomyosin subunits and in two species of $\operatorname{TnT}$ (labelled $\operatorname{TnT}_{1 \mathrm{r}}$ and $\mathrm{TnT}_{2 \mathrm{f}}$ ). This variation in thin filament regulatory components is not a function of fiber type $(8,33)$. In fibers from fasttwitch glycolytic muscles of rabbit two extremes of thin filament expression are observed. The 


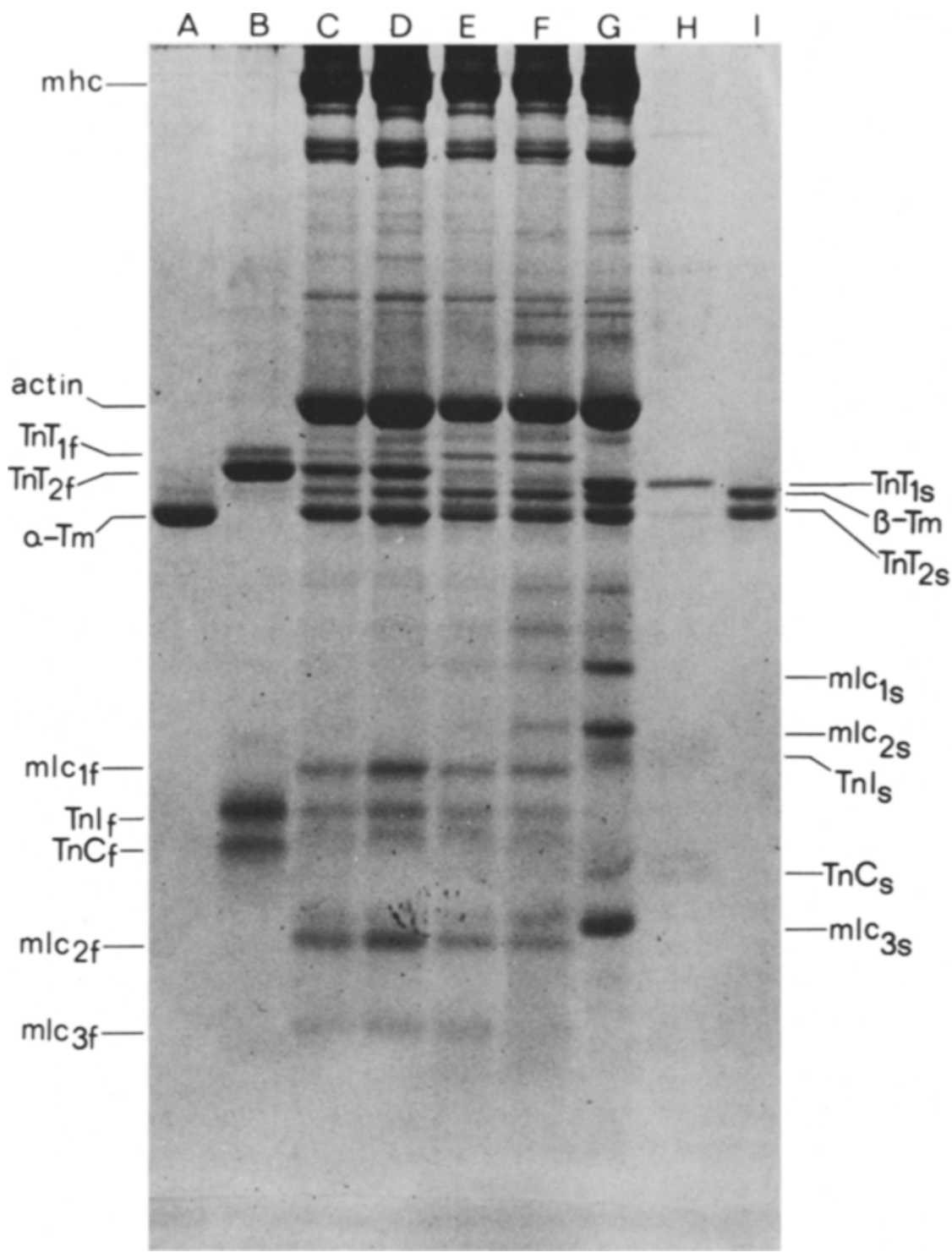

Figure 9. SDS-PAGE of myofibrillar preparations. $10.5 \%$ polyacrylamide gel electrophoresis in the presence of SDS of erector spinae (c), psoas (d), plantaris (e), diaphragm (f) and soleus (g) with fast tropomyosin (a) and troponin (b) purified erector spinae and slow troponin (h) and tropomyosin (i) from soleus as standards. The fast and slow myosin light chain identification is based on previous reports (34).

first, typical of back muscles erector spinae and psoas (Figure 9, lanes $\mathrm{C}$ and $\mathrm{D}$, respectively) is characterized by the expression of $\mathrm{TnT}_{2 \mathrm{f}}$ and the $(\alpha)_{2}$ tropomyosin dimer. The second, prevalent in the hindlimb muscles, tibialis and plantaris (Figure 9, lane E) is characterized by $\operatorname{TnT}_{1 f}$ and the $(\alpha \beta)$ tropomyosin dimer. To demonstrate that $\operatorname{Tn}_{1 \mathrm{f}}$ and $\operatorname{Tn}_{2 \mathrm{f}}$ are specific for fast muscles, a slow muscle, soleus, is shown in lane G. Diaphragm, which contains a mixture of fast and slow fibers, therefore, a mixture of proteins is in lane $F$.

The slopes of the $\mathrm{pCa} /$ tension relation of different types of fast fibers (reflected in $n_{H}$ ) are not all the same (Table II). Fibers from the psoas have a mean $n_{H}$ of 5.3, while fibers from the 
EDL and plantaris consistently have $n_{H}$ 's between 3 and 4 . Because we have not yet characterized the myosins from these fast-twitch glycolytic muscles, it would be premature to assert that these differences in slope are solely a consequence of differences in troponin $\mathrm{T}$ and tropomyosin expression. However, it is clear that high slopes correlate with the expression of $\operatorname{Tn} T_{2 r}$ and $(\alpha)_{2}$ tropomyosin and lower slopes with the expression of $\mathrm{TnT}_{\text {If }}$ and ( $\alpha$ ) tropomyosin.

\section{DISCUSSION}

The demonstration that extraction of an average of one $\mathrm{TnC}$ per regulatory strand leads to a decrease in $n_{H}$ (Figure 8 ) relies on two important assumptions. First, we rely on tension change to quantify the $\%$ TnC extracted and not on direct analysis of $\mathrm{TnC}$ loss. Tension change and the data of Table I does place an upper limit on the $\%$ extraction and for a $5 \%$ or less tension loss we can assume that no more than $5 \% \mathrm{TnC}$ is lost. There is no exact way to put a lower limit on the amount of TnC extracted, on average per strand, because it might be true that less than one statistically degrades the $\mathrm{pCa}$ /tension curve slope. This introduces our second assumption, that the extraction is uniform or random. We are, however, obtaining a collective response from millions of thin filaments at a time and do not know the exact distribution of the gaps. We can imagine, for example, two limits on a distribution equivalent to one TnC per regulatory strand. At one limit extraction is limited to the outer annulus which becomes so depleted that no calcium activated tension is generated in this zone. The rest of the fiber is intact and no change in slope is observed. At the other limit the extraction is greater towards the outer annuli and proportionately decreases towards the core. This case is about the same as assuming a random distribution of gaps on the strands except that the gaps are located more commonly towards the outside, i.e. $50 \%$ of the strands have two defects, $25 \%$ have one and $25 \%$ have none. We have done a number of computer simulations and the most illuminating one is the possibility that the outer annulus is about $50 \%$ extracted and this portion generates low slopes which sum with the normal slope of the rest of the fiber. The simulated $\mathrm{pCa}$ /tension curves coincide with control $\mathrm{pCa}$ /tension curves up to about $70 \%$ maximum tension while curves from extracted fibers diverge from control curves beginning at the foot. We believe, therefore, that the gaps are normally distributed among the population of regulatory strands.

Cooperativity in the thin filament control mechanism was originally proposed by BREMEL and WEBER (6) who reported that synthetic thin filaments activate hydrolysis of substrate (MgATP) by myosin heads ( $\mathrm{S} 1$ ) in a cooperative way dependent on the actin: S1 ratio. They suggested that binding of $\mathrm{S} 1$ to the thin filaments turns on the regulatory unit and enhances its affinity for calcium. The proposal that bound S1 turns on a regulatory unit is supported by the finding that N-ethylmaleimide treated $\mathrm{S} 1$ (this binds irreversibly to actin even in the presence of substrate) activates regulated actin strands $(27,30)$. GREENE and EISENBERG (16) found that $S 1$ bind cooperatively to regulated actin and they note that their model of cooperativity can be made to fit $\mathrm{Sl}$ binding data, collected in the absence of $\mathrm{Ca}^{2+}$ and substrate, better if the number of cooperating actins is increased beyond 7. In the presence of $\mathrm{Ca}^{2+}$ or substrate, the very high cooperativity of $\mathrm{S} 1$ binding to regulated actin strands nearly disappears (17), in contrast to our results on skinned fibers where the slope of the $\mathrm{pCa} /$ tension curve increases with increase in substrate (4).

Although we are the first to demonstrate that cooperativity unifies the thin filament, near neighbor cooperativity has been incorporated into models of S1 binding to actin strands (19, 20). A test for cooperative linkage between adjacent tropomyosin molecules was made by TRUEBLOOD et al. (36). Their experiments, however, seem to have excluded the overlap between adjacent tropomyosins from the mechanism. Our studies show that the removal of only $\mathrm{TnC}$ subunits reduces cooperativity, so, whatever else links neighbor units, it must include TnC. Our results also indicate that differences in troponin $\mathrm{T}$ and tropomyosin modulate the cooperativity.

The extensive cooperativity we find in the activation of the thin filament allows the 
dependence of the slope of the $\mathrm{pCa} /$ tension relation on sarcomere length (26) to be interpreted in another way than an effect on calcium binding. Partial population of only one end of the thin filament with attached myosin may activate a cooperative response which is weaker than that induced by an equivalent population of the entire length of the thin filament.

The $\mathrm{pCa}$ /tension curve is necessarily evidence that binding $\mathrm{Ca}^{2+}$ to the regulatory unit governs the tension process. If, as noted above, bound myosin also activates a regulatory unit, one must wonder how regulation stays fully controlled by $\mathrm{Ca}^{2+}$ ? If we accept, simply for discussion, that an $\mathrm{n}_{\mathrm{H}}$ of 8 means that $8 \mathrm{Ca}^{2+}$ must be bound somewhere along the thin filament for it to become active irrespective of the bound myosin, then the filament remains fully $\mathrm{Ca}^{2+}$ regulated. It may be possible to construct models in which the actin cofactor activity, once turned on by bound $\mathrm{Ca}^{2+}$, is modulated by the bound myosin without reducing control by $\mathrm{Ca}^{2+}$. Such a model fails, however, to explain activation by low substrate in the absence of $\mathrm{Ca}^{2+}(1,14,24,32$, 38). This remains the essential dilemma of dual control by $\mathrm{Ca}^{2+}$ and substrate, complicated by the present finding that the thin filament is controlled as a unit.

\section{ACKNOWLEDGEMENTS}

Supported by grants NIH NS-18228 (Dr. SCHACHAT) and the Muscular Dystrophy Association of America and NIH NS-1 17660 (Dr. BRANDT).

I thank HEINZ HoLTER for the sabbatical year (1978-79) I spent in the Physiological Department, Carlsberg Laboratory. This experience was at once stimulating yet tranquil and the memory of it becomes more precious with each passing year. I trace the origins of many of my present ideas on muscle function and my values about science and society to that year of work, reflection, and conversations with Heinz and the other members of the Department. P.W. BRANDT.

\section{REFERENCES}

1. BrandT, P.W. J.P. Reuben, H. GRundfest: Regulation of tension in the skinned crayfish muscle fiber. II. Role of calcium. J. Gen. Physiol. 59, 305-317 (1972)

2. BRandT.P.W., E. Chappel \& B.R. Jewel: A robust transducer suitable for measuring forces of $1 \mu \mathrm{N}$. J. Physiol., London 258, 43-44 (1976)

3. Brandt, P.W. R.N. Cox \& M. Kawal: Can the binding of $\mathrm{Ca}^{2+}$ to two regulatory sites on troponin$\mathrm{C}$ determine the steeps $\mathrm{pCa} /$ tension relationship of skeletal muscle?Proc. Natl. Acad. Sci. USA 77, 4717-4720 (1980)

4. BRandT, P.W., R.N. Cox. M. KaWal \& T. RobinSON: Regulation of tension in skinned muscle fibers: Effect of cross-bridge kinetics on apparent $\mathrm{Ca}^{2+}$ sensitivity. J. Gen. Physiol. 79, 997-1016 (1982)

5. Brandt. P.W., B. Gluck. M. Mini \& C.E. Cerri: Regulation of tension in skinned muscle fibers: Hysteresis of the mammalian $\mathrm{pCa}$ /tension relation is small and muscle specific. Submitted

6. BREMEL. R.D. \& A. Weber: Cooperation within actin filament in vertebrate skeletal muscle. Nature New Biol. 238, 97-101 (1972)

7. BRIGGS, M.M. \& F.H. SCHACHAT: Heterogeneity of thin filament calcium regulatory proteins of rabbit skeletal muscle. Biophysical J. 41, 297a (1983)

8. BRonson. D.D. \& F.H. SChaChat: Heterogeneity of contractile proteins: Differences in tropomyosin from fast, slow, and mixed skeletal muscles of the rabbit. J. Biol. Chem. 257, 3937-3945 (1982)

9. Chalovich. J.M. \& E. EisenberG: Inhibition of actomyosin ATPase activity by troponin-tropomyosin without blocking the binding of myosin to actin. J. Biol. Chem. 257(5), 2432-2437 (1982)

10. Chantler. P.: Retreats from the steric blocking of muscle-contraction. Nature 298(5870), 120-121 (1982)

11. Cox, J.A., M. Comte \& E.A. Stein: Calmodulinfree skeletal muscle troponin $C$ prepared in the absence of urea. Biochem. J. 195, 205-211 (1981)

12. Eastwood, A.B.. D.S. Wood, K.L. BocK \& M.M. SORENSON: Chemically skinned mammalian skeletal muscle. I. The structure of skinned rabbit psoas. Tissue \& Cell 11, 553-566 (1979)

13. EisenberG, E. \& W.W. Kielley: Troponin-tropomyosin complex. Column chromatographic separation and activity of the three, active troponin components with and without tropomyosin present. J. Biol. Chem. 249(15), 4742-4748 (1974)

14. Ferenzi, M.A., R.M. Simmons \& J.A. Sleep: General considerations of cross-bridge models in relation to the dependence on MgATP concentration or mechanical parameters of skinned fibers from frog. In Basic biology of muscle: A comparative approach. B.M. Twarog, R.J.C. Levine \& 
M. Dewey Eds. Raven Press, N.Y., USA, pp. 223-243 (1982)

15.FLICKER, P.F., G.N. Phillips \& C. COHEN: Troponin and its interactions with tropomyosin: An electron microscope study. J. Mol. Biol. 162, 495-501 (1982)

16. Greene, L.E. \& E. Eisenberg: Cooperative binding of myosin subfragment-1 to the actin-troponin-tropomyosin complex. Proc. Nat. Aca. Sci. USA 77, 2616-2620 (1980)

17. Greene, $L$ : The effect of nucleotide on the binding of myosin subfragment 1 to regulated actin. J. Biol. Chem. 257, 13993-13999 (1982)

18. Hazelgrove, J.C.: X-ray evidence for a comformational change in the actin-containing filaments of vertebrate striated muscle. Cold Spring Harbor Symp. Quan Biol. 37, 341-359 (1972)

19. HiLL, T.L. E. EISENBERG \& L. GreENE: Theoretical model for the cooperative equilibrium binding of myosin subfragment 1 to the actin-troponintropomyosin complex. Proc. Natl. Acad. Aci. USA 77, 3186-3190 (1980)

20. HiLl, T.L., E. EISENBERG \& L.E. GREENE: Alternate model for the cooperative equilibrium binding of myosin subfragment-1-nucleotide complex to actin-troponin-tropomyosin. Proc. Natl. Acad. Sci. USA 80(1), 60-64 (1983)

21. HUXLEY.H.E.: Structural changes in the actin- and myosin-containing filaments during contraction. Cold Spring Harbor Symp. Quan Biol. 37, 361-376 (1972)

22. KaWAi.M. \& M. ORentlicher: Effect of inorganic phosphate $\left(\mathrm{P}_{\mathrm{i}}\right)$, substrate (MgATP), and excess ATP on complex stiffness of skinned crayfish muscle fibers and glycerinated rabbit psoas muscle bundles. Biophysical J. 16, 152a (1976)

23. LAEMMLI, U.K.: Cleavage of structural proteins during the assembly of the head of bacteriophage T4. Nature 227(259), 680-685 (1970)

24. LoXdale, H.D. \& R.T. TREGEAR: Generation of tension by glycerol-extracted vertebrate skeletal fibres in the absence of calcium. J. Muscle Research \& Cell Motility 4, 543-556 (1983)

25. Moss, R.L., G.G. Giulian \& M.L. Greaser: Effects of EDTA treatment upon the protein subunit composition and mechanical properties of mammalian single skeletal muscle fibers. J. Cell Biol. 96, 970-978 (1983a)

26. Moss, R.L., A.E. SWINFORD \& M.L. GREASER: Alterations in the $\mathrm{Ca}^{2+}$ sensitivity of tension development by single skeletal muscle fibers at stretched lengths. Biophysical J. 43, 115-120 (1983b)

27. Nagashima, H. \& S. ASakura: Studies on cooperative properties of tropomyosin-actin and tropo- myosin-troponin-actin complexes by the use of $\mathrm{N}$-ethylmaleimide treated and untreated species of myosin subfragment-1. J. Mol. Biol. 155, 409428 (1982)

28. Orentlicher. M., J.P. Reuben. H. Grundfest \& P.W. BrandT: Calcium binding and tension development in detergent-treated muscle fibers. J. Gen. Physiol. 63, 168-186 (1974)

29. PARRY. D.A.D. \& J.M. SQuires: Structural role of tropomyosin in muscle regulation. Analysis of the $\mathrm{X}$-ray patterns from relaxed and contracting muscle. J. Mol. Biol. 37, 251-262 (1973)

30. Pemrick. S.\&A. Weber: Mechanism of inhibition of relaxation by N-ethylmaleimide treatment of myosin. Biochemistry 15, 5193-5198 (1976)

31. Potter, J.D. \& J. Gergely: The calcium and magnesium binding sites on troponin and their role in the regulation of myofibrillar adenosine triphosphatase. J. Biol. Chem. 250, 4628-4633 (1975)

32. Reuben, J.P., P.W. Brandt, M. Berman \& H GRUNDFEST: Regulation of tension in the skinned crayfish muscle fiber. I. Contraction and relaxation in the absence of $\mathrm{Ca}(\mathrm{pCa}>9)$. J. Gen. Physiol. 57, 385-407 (1971)

33. Schachat, F.H., A.D. Magid, D.D. Bronson \& O.B. MCDONALD: Gene expression in single muscle fibers from rabbit skeletal muscles. J. Cell Biol. 87, 263a (1980)

34. Schachat, F.H., D.D. Bronson \& O.B. MCDoNALD: Two different kinds of SO muscle fibers which differ in their myosin light chain complements. FEBS lett. 122, 80-82 (1980)

35. Sobieszek, A.: Steady-state kinetic studies on the actin activation of skeletal-muscle heavy-meromyosin subfragments - effects of skeletal, smooth and non-muscle tropomyosins. J. Mol. Biol. 157(2), 275-286 (1982)

36. Trueblood, C.E., T.P. Walsh \& A. Weber: Is the steric model of tropomyosin actin valid? In: Basic Biology of Muscle: A comparative approach. B.M. Twarog, R.J.C. Levine \& M. Dewey Eds. Ravel Press, N.Y. USA, pp. 223-243 (1982)

37. WALSH, T.P. \& WEGNER, A.: Effect of the state of cysteine 190 of tropomyosin on the assembly of the actin-tropomyosin complex. Biochim. Biophys. Acta $626,79-87$ (1980)

38. Weber, A.: Parallel response of myofibrillar contraction and relaxation to four different nucleotide triphosphates. J. Gen. Physiol. 55, 781-791 (1969)

39. Wood, D.S., J. Zollman, J.P. Reuben \& P.W. BRANDT: Human skeletal muscle: properties of the "chemically skinned" fiber. Science 187,1075 1076 (1975) 